\title{
Network Systems Modelled by Complex Cellular Automata Paradigm
}

\author{
Pawel Topa \\ AGH University of Science and Technology \\ Poland
}

\section{Introduction}

Most historical sources say that Cellular Automata were discovered by John von Neumann and Stanislaw Ulam in the forties of the twentieth century. Von Neumann was investigating the problem of self-reproducing systems in biology. His initial kinematic model appeared to be too complicated and he followed the suggestion from Stanislaw Ulam, who at the same time worked on problems of crystal growth. Von Neumann and Ulam defined an abstract universe in the form of two-dimensional regular mesh with interacting entities Neumann (1966).

In the seventies mathematician John Conway published his set of rules called "Game of Life". His work was popularized by Martin Gardner in the pages of Scientific American. The rules of "Game of Life" implemented on computers showed an amazing world of living and dying automata. Its dynamics appeared to be so surprisingly complex that it became area of intensive studies. Vast amount of work concentrated on searching for initial conditions that evolve into non-trivial behaviour of Cellular Automata. One of the most important achievement was proving that "Game of Life" can simulate Universal Turing Machine i.e. can act as an abstract model of any computer.

In the eighties Stephen Wolfram spent part of his scientific career on investigating properties of one-dimensional Cellular Automata. His important achievement was definition of 256 rules for one-dimensional Cellular Automata. He also introduced first classification scheme based on long period observation. The crowning achievement of his works was a book "A New kind of Science" Wolfram (2002). In this monumental work Wolfram outlines a new paradigm for modelling complex phenomena with Cellular Automata approach.

Scientific research related to Cellular Automata were not only limited to abstract mathematical "toy". Toffoli, Margoulus and Fredkin postulated application of cellular automata for modelling physical systems Chopard \& Droz (1998); Toffoli \& Margolus (1987). They noticed that physical laws can be encoded in rules of interaction in temporally and spatially discrete universe.

The milestone in Cellular Automata theory was the lattice gas model HPP developed by Hardy et al. (1976). The model, which is in fact the Cellular Automata, consists of a simple and fully discrete system of moving and colliding particles. Although, the idea of lattice gas automata was invented independently, the Cellular Automata theory provided clear conceptual framework. HPP model and his successor FHP Frisch et al. (1986) was successful in modelling complex phenomena as flows in porous media, spreading of a liquid droplet and wetting phenomena.

It is difficult now to figure out all the areas where the Cellular Automata paradigm is applied: 
- biological processes: tissue growth Wang et al. (2008), angiogenesis Markus et al. (1999), cancer development Reis et al. (2009) etc.

- chemical processes Chopard \& Droz (1998); Kier (2000); Kier et al. (1998),

- granular flow Baxter \& Behringer (1991); Masselot \& Chopard (1996),

- pedestrian dynamics and traffic flow Blue et al. (1997); Was (2005),

- geological processes D’Ambrosio et al. (2001).

"Cellular Automata Modelling of Physical Systems" by Chopard \& Droz (1998) contains very profound review of various applications of Cellular Automata approach for modelling physical systems.

Cellular Automata in their classic meanings have rather limited application area. On the other hand their simplicity in computer implementation is irresistible temptation. A lot of extensions were introduced to their definition. The set of states was extended by introducing floating point numbers instead of logical values. The neighbourhood notion was extended by introducing new schema (von Neumann, Moore and Margoulus neighbourhoods) and range bigger then one cell. The homogeneity of Cellular Automata were also broken. In some applications synchronous updating were also replaced by other asynchronous schemas. Mesh types other then rectangular were also applied in some models. Some extensions make that model is so far from classic Cellular Automata definition that it could be classified as a different modelling tool. The Cellular Potts model Graner \& Glazier (1992) and Agent-Base models Wooldridge (2009) can be treat as generalized Cellular Automata.

Cellular automata paradigm fits perfectly to paradigm of computation on digital computers. When we use Cellular Automata approach we do not have to struggle with round-off errors, truncation errors, numerical stability and lots of other problems that appear when we implement methods of numerical analysis on computers. Rules of local interaction can be easily and unambiguously defined by using programming languages constructions (if ... then ... , while ... do ...). Algorithms and data structures necessary to develop Cellular Automata models are relatively simple and give great opportunity to efficient implementation. Moreover, Cellular Automata are inherently parallel. Cellular Automata definition states that cells have to be processed simultaneously. Computer implementation have to simulate this by using additional temporary data structure. In the $80 \mathrm{~s}$ and $90 \mathrm{~s}$ of 20 th century a lot of work had been devoted to constructing hardware implementation of Cellular Automata. The most famous were Cellular Automata Machines built by Tommaso Toffoli and Norman Margoulus (CAM-6 i CAM-8) Margolus et al. (1986); Toffoli \& Margolus (1987). There were also successful attempts to implement CA on transputer machines Cannataro et al. (1995); Somers \& Rem (1989) however this architecture did not succeed an fallen into oblivion. Finally, these interesting researches were abandoned in favour of flexibility of programming solutions. Recent research in GPGPU computing methods shows that Cellular Automata can run very effective on graphic processors Gobron et al. (2010).

In this chapter I present applications of Cellular Automata paradigm for modelling dynamically evolving system with networks structure that function as transportation pathways. The following section presents phenomena that was studied and modelled by the author. Next I present the general idea of Graph of Cellular Automata that combine of Cellular Automata and graphs. In further section I present two models that employed Graph Cellular Automata:

- model of anastomosing river system,

- model of tumour induced angiogenesis. 
I conclude the chapter with discussion on extensions and modifications that make Cellular Automata useful in modelling physical systems.

\section{Network systems modelled with cellular automata}

Cellular Automata fit best for modelling systems that are characterized by inner structural homogeneity and regularity, for example crowd of pedestrians or fluids. When we look for Cellular Automata that are able to capture complex systems with interacting parts that have a different structure, we have to extended classic definition and combine it with other methods. One of the phenomena that we observe in everyday life are transportation systems located in consuming or producing environments. Transportation networks are transfer pathways for various substances which are produced or absorbed by surrounding environments. These substances can also diffuse through the environment. We can enumerate two examples of such the systems that we meet in everyday life:

- Vascular systems - blood vessels transport nutrients and oxygen which penetrate into the tissue and nourish it. Products of metabolism penetrate from tissue to blood vessels and they are removed from the body. Tissue can influence the vascular network by producing various substances called angiogenic factors that stimulate angiogenesis (process of formation of blood vessels).

- River systems - rivers transport organic and mineral materials from upper, erosional part of river system to lower part were the loads are deposited. In whole the basin, the surrounding environment (shape of terrain) are modified by river (erosion and deposition). Changes in terrains influence river systems by changing the routes of channels as well as the shape of network.

Such the systems are characterized by coexistence two parts having completely different structure. Environment has (in general) regular, uniform structure where substances are distributed or collected in whole area or volume. Regular mesh with dense network of connection between cells can accurately approximate these processes. In the networks, processes are rather limited to the paths defined by their branches. Thus, we can easily simplify their representation by using graph.

Graph of Cellular Automata is a hybrid modelling method employing Cellular Automata theory and some elements of graph theory. Cellular Automata is a basis that is used to construct graph. Some cells from mesh of automata are selected and they establish set of nodes in graph. An additional relations of neighbourhood are established between these cells, what constitute set of edges. Figure 1 illustrates this structure.

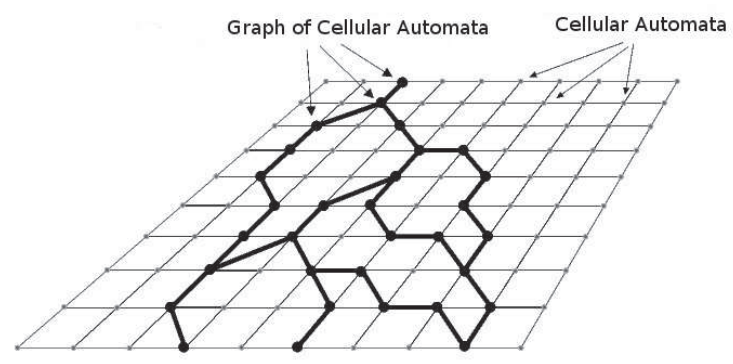

Fig. 1. Graph of Cellular Automata, from Topa \& Dzwinel (2009) 
Regular mesh and graph are processed separately and often in different manner (e.g. synchronously versus asynchronously). Cells that belong to the graph act as connections between these two systems and provide interaction between them. State of these cells are transfer way for results of environment simulation on regular mesh of Cellular Automata to the graph structure representing network system (see Fig. 2).

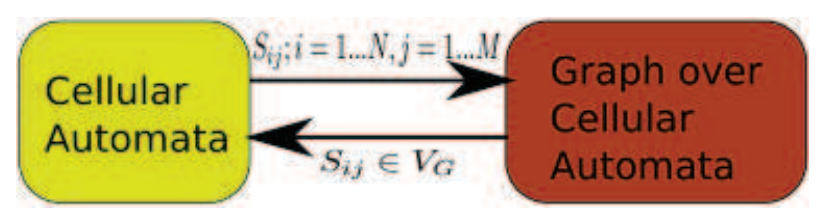

Fig. 2. Interaction between Cellular Automata and Graph constructed over Cellular Automata is transfered through the states of cells. States of all the cells in the mesh $\left(S_{i j}\right)$ can influence evolution of network system encoded in graph. Network system influences environment through the cells that belong to graph $\left(S_{i j} \in V_{G}\right)$

The paradigm of Graph of Cellular Automata was applied for simulating two phenomena:

- Anastomosing river systems Topa \& Dzwinel (2003); Topa et al. (2006); Topa \& Paszkowski (2001)

- Tumour Induced Angiogenesis Topa (2006; 2008); Topa \& Dzwinel (2009)

\subsection{Rivers and anastomosing river systems}

Anastomosing rivers are interesting dynamic system created as a result of interaction between constructive and destructive processes. Anastomosing systems are formed in middle part of river system. Slope on these area is very small - approximately $10 \mathrm{~cm}$ per $1 \mathrm{~km}$. Flow rate is also very small, river do not erode terrain. Organic and non-organic materials carried by river are mostly transported through this part of river and deposited later in lower reaches. Waters rich of nutrients penetrate soil in surrounding banks. Fertile areas near river channels are characterized by a significant increase in vegetation. Products of plant decay deposit on this area as peat (approximately 1-1.5 mm/year Gradzinski et al. (2003)). Peat accumulation lasting for thousands years may produce a peat bog with a depth of several meters.

The processes that occur on river channel banks are crucial for creation anastomosing system. Figure 3 illustrates these processes. It presents cross-section of river channel. The rate of peat accumulation depends on the distance from river channel. Water penetrates soil nearby the channel and supplies nutrients. Nutrients are gathered by plants that vegetate on the river banks. On the areas that are more distant from water, the vegetation are less abundant than on the areas that directly neighbour to channel. Consequently, the rate of peat deposition decreases with distance from channel. Deposition on river banks is accompanied by deposition on river bottoms. As a result the whole river channel is raised (see in Fig 3). The water level is raised above the average valley ground level. Sometimes channel can be also partly blocked by clusters of floating plants, tree trunks etc. It is metastable state which can be easily destroyed. High levels of water that usually occur during spring floods can cause breaking river banks above obstacles. River can create bypass channel that evade narrowness on primary channel. The shape of terrain in valley usually force new channel to join primary channel somewhere downstream.

The routes of new channels run through local depression areas. Evolution of new channel proceed in the same way as the parent channel. Old channels may also disappear gradually. The system evolves to dynamic metastable state in which whole the area of the valley is 


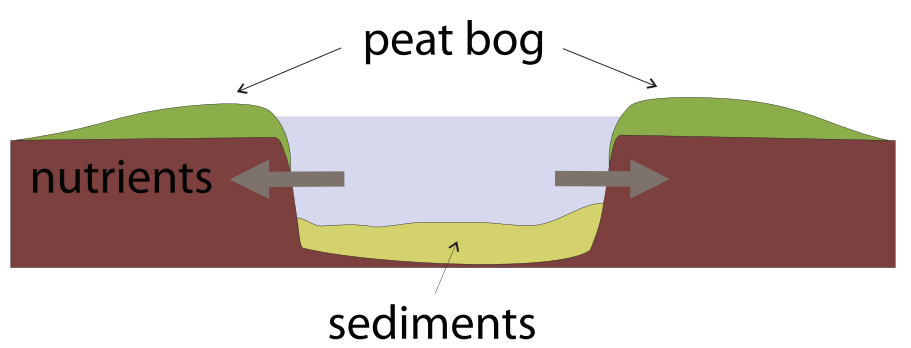

Fig. 3. Crossection of anastomosing river channel

equally supplied by river systems. As a result the bottom of valley raises covered by thick (several meters) layer of peat-bog.

Anastomosing rivers create very complex pattern of forking and joining channels. Fig. 4 presents part of real network of river Narew located in eastern Poland (Central Europe). It is relatively small anastomosing river comparing to huge anastomosing systems created by river $\mathrm{Ob}$ in Syberia (anastomising system can be observed from space) and Okawango in Africa.

2.2 Vascular system and tumour-induced angiogenesis

Angiogenesis is the process of formation of vascular network Carmeliet (2005). Physiologically this process occurs during embryogenesis. In mature organisms it often accompanies to cancer development and this pathological process is called tumour-induced angiogenesis. Inhibition of tumour induced angiogenesis is one of the treatments applied in oncology.

Tumour growth is a multi-stage and multi-scale process that starts with the loss of control of cell proliferation Carmeliet (2005). Initially, tumour is in avascular state and draws nutrients from surrounding vessels. At this stage cluster of tumour cells can reach size aproximately 1 - $3 \mathrm{~mm}$. Tumour remains in steady state with balance between dying and proliferating cells. Without blood vessels, tumours cannot grow beyond a critical size and invade other regions of body. Tumour induced angiogenesis starts when the production of pro-angiogenic factors overcomes other forces that kept the angiogenesis quiescent so far.

Oxygen and nutrients penetrate the tissue only in a certain distance from the vessel. Distant cells, influenced by metabolic stresses, synthesise several angiogenic stimulators including VEGF (Vascular Endothelial Growth Factor) and PDGF-BB (Platelet-derived growth factor), Ang2 and NOS (Nitric Oxide Synthase) Carmeliet (2005); Steve et al. (2004). Stimulators migrate towards the nearest blood vessels. When they reach vessel, the endothelial cells (ECs) that lines the wall of this vessel are activated. They start to proliferate and migrate towards the tumour cells attracted by VEGF and other stimulators. The wall of the parent blood vessel becomes degraded and it opens to a new capillary. Migrating and proliferating ECs form a hollow tube-like cavity (the lumen), which are stabilised later by smooth muscle cells and perycites.

There are many differences between normal and tumour induced angiogenesis. Temporal and spatial expression of angiogenic factors are not well coordinated, what follows to non-uniform vascular development. As a result, new vessels form a highly chaotic and disorganised network Tonini et al. (2003). Tumour vessels are deprived various protective mechanisms i.e. perivascular cells that protect vessels from changes in oxygen or hormonal balance. Moreover the lumen does not have proper construction. Their walls may be partially constructed from cancer cells. They have also pathological form, e.g. they are thin and permeable, their diameter 


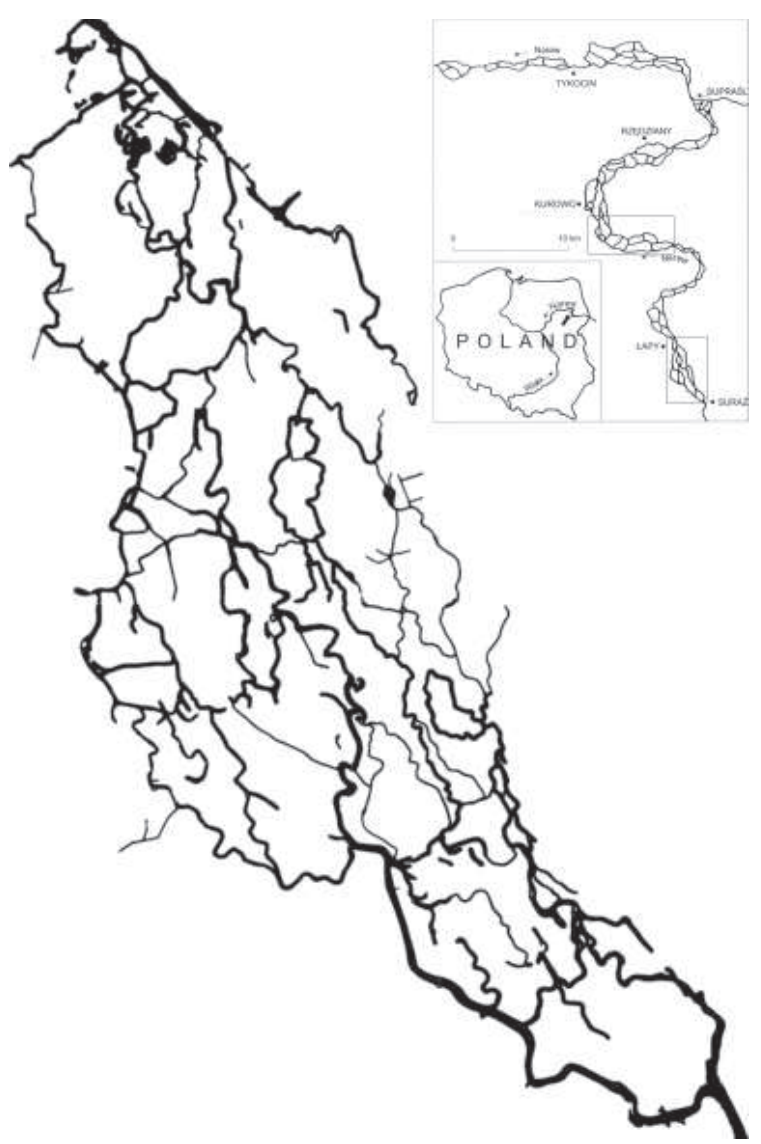

Fig. 4. Pattern of river channels in anastomosing part of Polish river Narew, from Topa et al. (2006)

changes abruptly. Proliferating cancer cells can also crash the vessels. All it makes that newly formed capillaries are still unable to supply starving tumour cells with oxygen and nutrients. Inhibition of tumour-induced angiogenesis is one of the most promising strategy in anti-cancer therapy Carmeliet (2005); Ferrara \& Kerbel (2005). Tumour cells deprived of nutrients and oxygen undergo necrosis. Moreover, the lack of vascular system prevents cancer cells from invading to other tissues (there is no routes, which tumour cells can use to transfer to other part of body). However, clinical tests show that none of the tested inhibitors succeed in broad range types of cancers Carmeliet (2005). Monotherapies fail because angiogenesis is controlled by very complex balance of stimulators and inhibitors. Therefore, further investigations have to concentrate on studies including wider range of angiogenic factors.

\section{The models}

The Graph of Cellular Automata modelling tool was initially invented for modelling anastomosing river systems Topa \& Paszkowski (2001). Pure Cellular Automata model appeared too computationally demanding and it could not reflect global evolution of the 

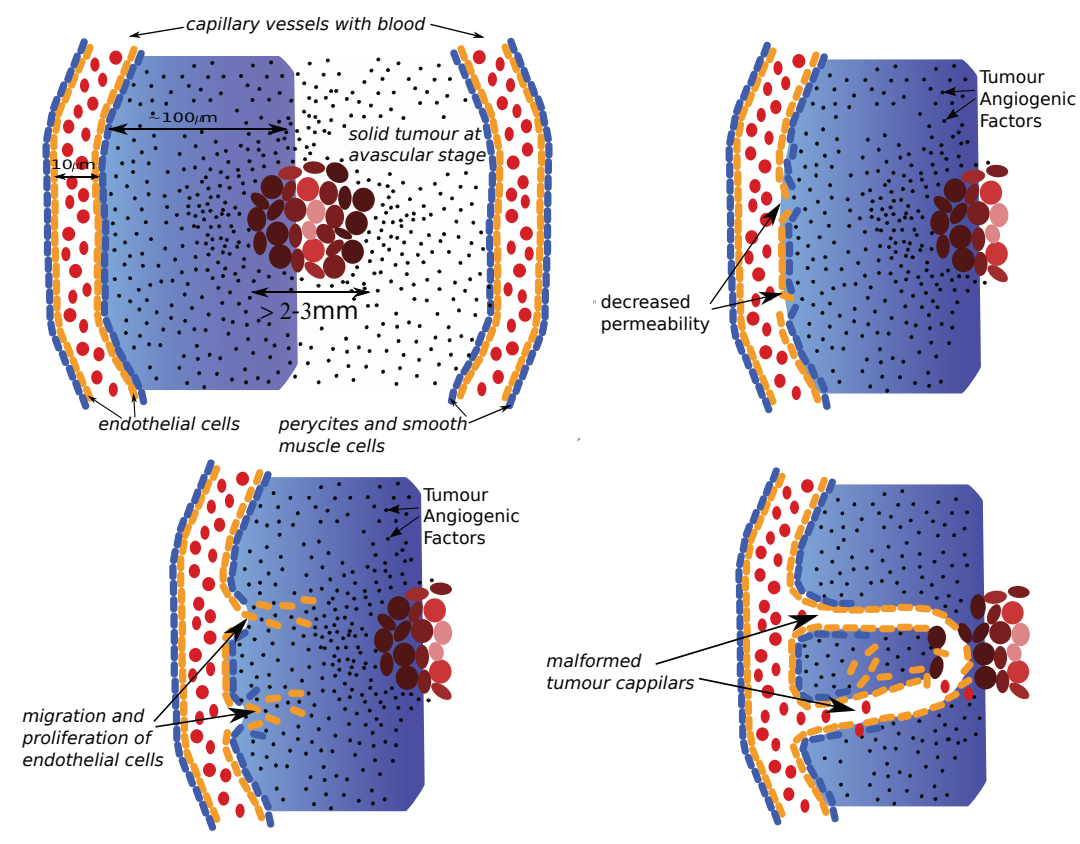

Fig. 5. Stages of tumour induced angiogenesis

system Topa et al. (2006). The model called MANGraCA (Model of Anastomosing Network with Graph of Cellular Automata) is constructed in the following way:

Cellular Automata is applied to model peat bog in the river valley and the following processes are reflected in this part of the model:

- nutrient distribution,

- growth of peat layer.

Graph of Cellular Automata is applied to model network of river channels. The following processes are implemented in this module:

- flow in river channels,

- decreasing of channel throughputs caused by peat bog development and sedimentation,

- branching, routing of new channels, anastomosing.

Cells that belong to the graph are the source of nutrients from where they are distributed over the regular mesh of automata that represent peat bog.

In more formal way the model can be defined as follows:

$$
C A_{M A N G r a C A}=\left\langle Z^{2}, X_{K}, G_{C A}, S, \delta_{K}, \delta_{G}\right\rangle
$$

- $Z^{2}$ - a collection of cells ordered as a square or hexadecimal mesh of $Z \times Z$ cells,

- $X_{K}-$ neighbourhood for the $(i, j)$ cell in the regular mesh of automata (Moore neighbourhood is used in the model),

- $G_{C A}$ - a planar, directed and acyclic graph defined as $\left(V_{G}, E_{G}\right)$, where $V_{G} \subset Z^{2}$ and $E_{G} \subset Z^{2} \times Z^{2}$ are finite sets of vertices and edges, respectively, 
- $S$ - is the set of state vectors corresponding to each cell: $S=S_{m} \times S_{g}$ :

1. states for cells in regular mesh $S_{m}=(g, n, p)$ :

(a) $g$ - initial altitude,

(b) $p$ - thickness of peat layer,

(c) $n$ - nutrients density,

2. states for graph cells $S_{g}=(f, r)$ :

(a) $f$ - flow rate,

(b) $r$ - throughput;

- $\delta_{K}:\left(g^{t}, n^{t}, p^{t}\right) \rightarrow\left(g^{t+1}, n^{t+1}, p^{t+1}\right)$ - set of rules applied synchronously for each cell in $Z^{2}$.

- $\delta_{G}:\left(f^{t}, r^{t}\right) \rightarrow\left(f^{t+1}, r^{t+1}\right)$ - set of rules applied for each cell that belong to graph.

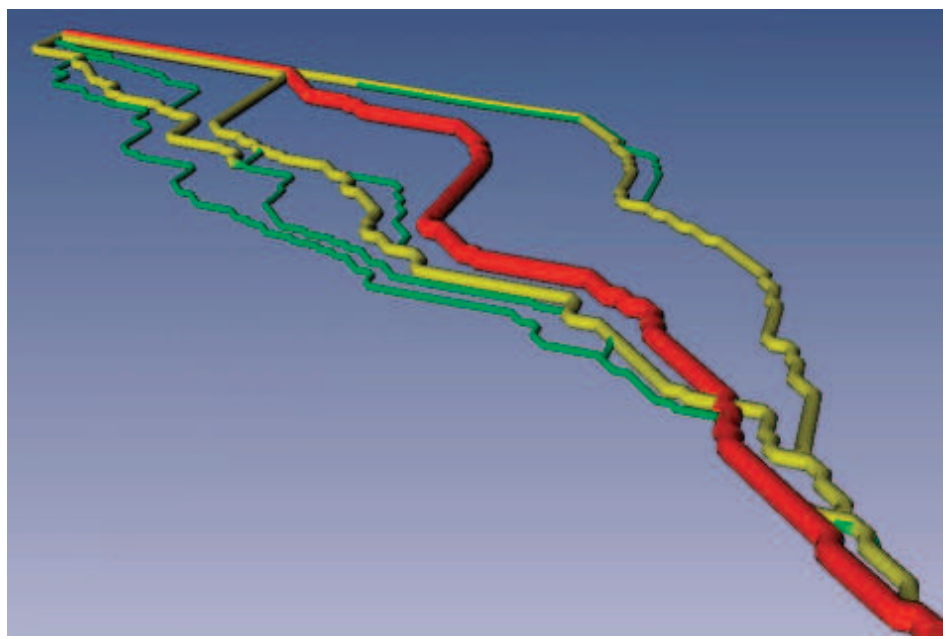

Fig. 6. Hierarchical structures of anastomosing pattern generated with model of anastomosing river.

The simulation program consists of two modules:

1. Cellular Automata Module - it calculates and update cells in regular mesh of cellular automata. It simultaneously apply defined rules of local interactions $\left(\delta_{K}\right)$ to each cell in the mesh and calculated new states.

- Nutrients density $\left(n_{i j}\right)$ is calculated as average density in neighbourhood. Cells that belong to graph have maximum density.

- Thickness of peat layer $\left(p_{i j}\right)$ is increased proportionally to the nutrients density.

2. Graph of Cellular Automata Module - it updates states of the cells that belong to the graph.

- Throughput $r_{i j}$ is decreased proportionally to thickness of peat bog layer $\left(p_{i j}\right)$ in neighbourhood with random modifier.

- Flow rate $f_{i j}$ is updated asynchronously starting from "root" node of the network. The flow in river channels was calculated by using simple algorithm: the amount of water that enters the node is equal to amount that leaves node. 
- Channel forming - the algorithm looks for cells with flow rate $f_{i j}$ less than throughput $r_{i j}$. Such the cells are a splitting point. The route of new channel is calculated based on altitude (initial altitude and thickness of peat layer).

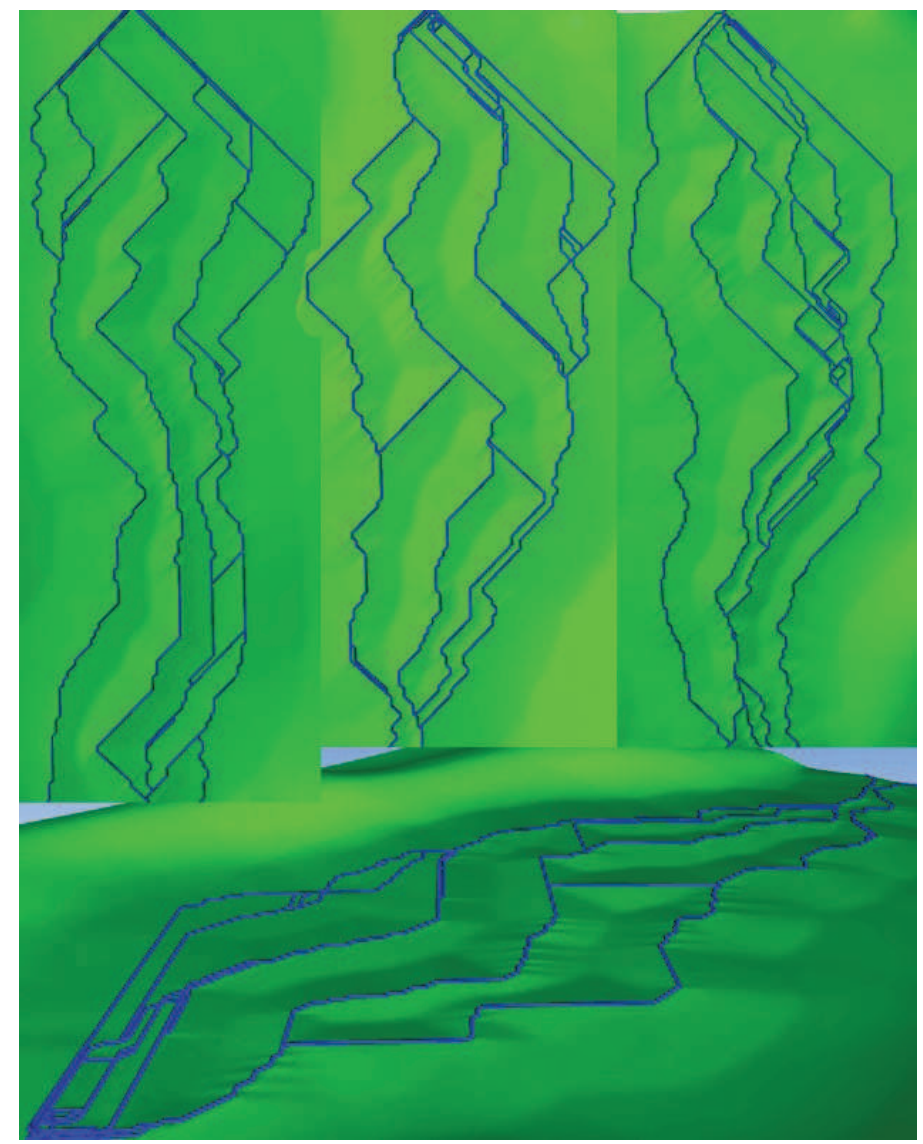

Fig. 7. Sample results of simulation anastomosing river with Graph of Cellular Automata paradigm.

The Graph of Cellular Automata was applied for modelling tumour induced angiogenesis with almost no changes in general structure Topa (2006; 2008); Topa \& Dzwinel (2009).

Cellular Automata model the tissue. Some cells are initially set to represent cancer cells. In this model cancer development processes were not included. Cancer cells do not multiply during the simulation. The cancer cells are sources of various tumour angiogenic factors (e.g. VEGF, PDGF-B, Angiopoeitin) that are distributed over the mesh.

Graph of Cellular Automata is constructed over the regular mesh of cellular automata and represents vascular systems. Cells that belong to graph are the source of nutrients and oxygen in regular the mesh of automata. These two substances are spread in mesh to the neighbouring cells. The cells that are nourished sufficiently stop producing tumour angiogenic factors. Graph that represents vascular systems develops in two ways: 
- growth at "tip" cells - cells in the graph are marked as "tip" when they are located at the end of sprout. During simulation algorithms checks the tip cells and if necessary conditions are fulfilled next neighbouring cells are added to the graph. The new cell are now the "tip" cell.

- branching - when the necessary conditions are fulfilled, cell that belong to graph can create branch (it cannot be a "tip" cell). The algorithm searches in its neighbourhood for cell that are added to graph and marked as "tip" cells for this branch. The development of the sprout proceed independently from parent vessels.

When growing vessel meets the other they join creating anastomosis.

Newly created vessels have to mature what means covering by perycites and smooth muscle cells. Only mature vessels can transport blood, supply nutrient to surrounding tissue and branch.

The model can be defined in a similar way as MANGraCA:

$$
C A_{A N G}=<Z^{n}, G_{C A}, X_{K}, S, \delta>\text { where : }
$$

- $Z^{n}$ - a collection of cells ordered as a square, hexadecimal $(n=2)$ or cubical $(n=3)$ mesh.

- $G_{C A}$ - directed and acyclic graph defined as $\left(V_{G}, E_{G}\right)$, where $V_{G} \subset Z^{n}$ and $E_{G} \subset Z^{n} \times Z^{n}$ are finite sets of vertices and edges, respectively,

- $X_{K}(i, j)$ - neighbourhood for the $(i, j)$ cell in the regular mesh of automata,

- $S$ - is the set of state vectors corresponding to each cell: $S=S_{m} \times S_{g}$,

- $S_{m}$ - represents the following states corresponding to all the cells in the regular CA mesh:

* $\quad t_{i j}$ - state of a single tumour cell, refers to its level of supplies with nutrients,

* $t a f_{i j}$ - TAFs concentration,

* $n_{i j}$ - nutrient (oxygen) concentration,

* per $_{i j}$ - perycites and smooth muscle cells concentration,

* $a a f_{i j}$ - AAF (anti-angiogenic factors) concentration.

- $S_{g}$ - represents states corresponding to the cells that belong to the Graph of Cellular Automata:

* age $e_{i j}$ — "age", maturation level,

* tip $p_{i j}$ - indicate "tip" cell (boolean),

* pres $_{i j}$ - pressure value,

* flow flj $_{i j}$ flow value;

The model is implemented in similar manner as the model of anastomosing river. It consist of two modules, one for calculation on regular mesh of automata and second that process graph structure:

Cellular Automata : some cells are marked as tumour cells and they are the sources of TAF

(Tumour Angiogenic Factors). Cells that belong to graph act as sources of nutrients. The following processes are modelled in this module:

- TAF distribution,

- nutrients distribution - tumour cells that are supplied with nutrients stop producing TAF, 


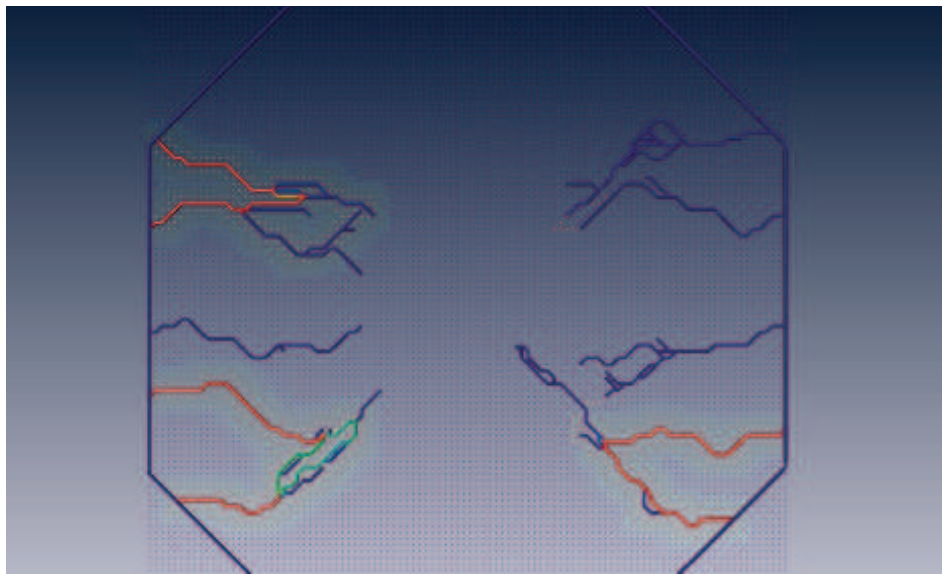

Fig. 8. Configuration with two primary vessels and cancer cells located in the center of area. Vessels are coloured according to flow value - blue means no flow, while red reflect maximum flow.

- distribution of other substances that can be included to the model (angiogenic inhibitors, perycites etc.)

Graph of Cellular Automata consists of the following processes:

- updating maturation level,

- calculating flows in vessels,

- developing sprouts - for each "tip" cells in the graph their successor is calculated,

- creating new sprouts - new sprout is created in mature cells with some probability (other conditions can be also included),

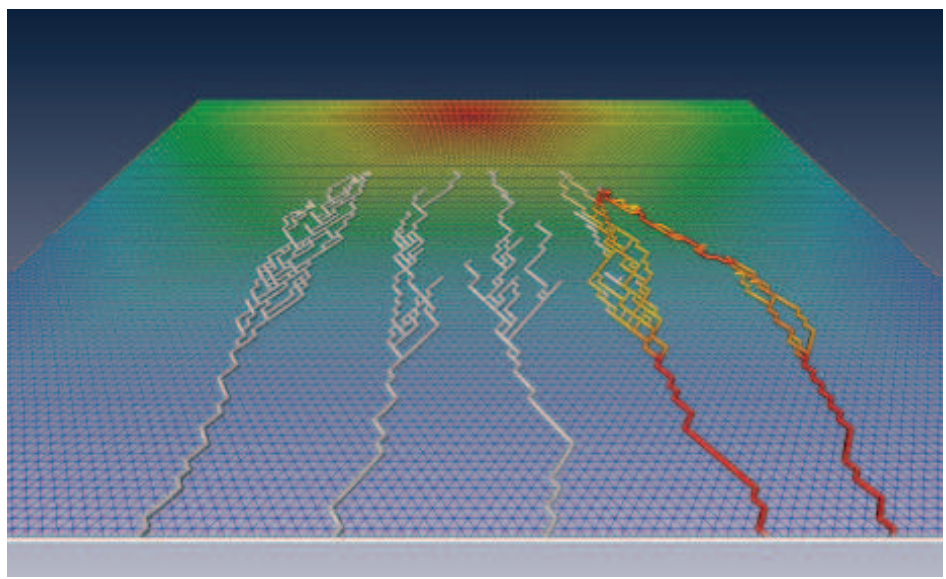

Fig. 9. Hexagonal mesh can be also applied instead of rectangular. Network segments are coloured according to flow value. 
Vascular system represented by graph structure allows for convenient blood flow calculation. The model uses Poiseuille equation for calculating flow in vascular segments Mcdougall et al. (2002).

$$
Q_{i j}=\frac{\pi R_{i j}^{4} \Delta P_{i j}}{8 \mu L_{i j}}
$$

- $R_{i j}$ - segment diameter,

- $L_{i j}$ - segment length,

- $\mu$ - viscosity,

- $\Delta P_{i j}=P_{i}-P j-$ pressure difference between nodes $i$ and $j$.

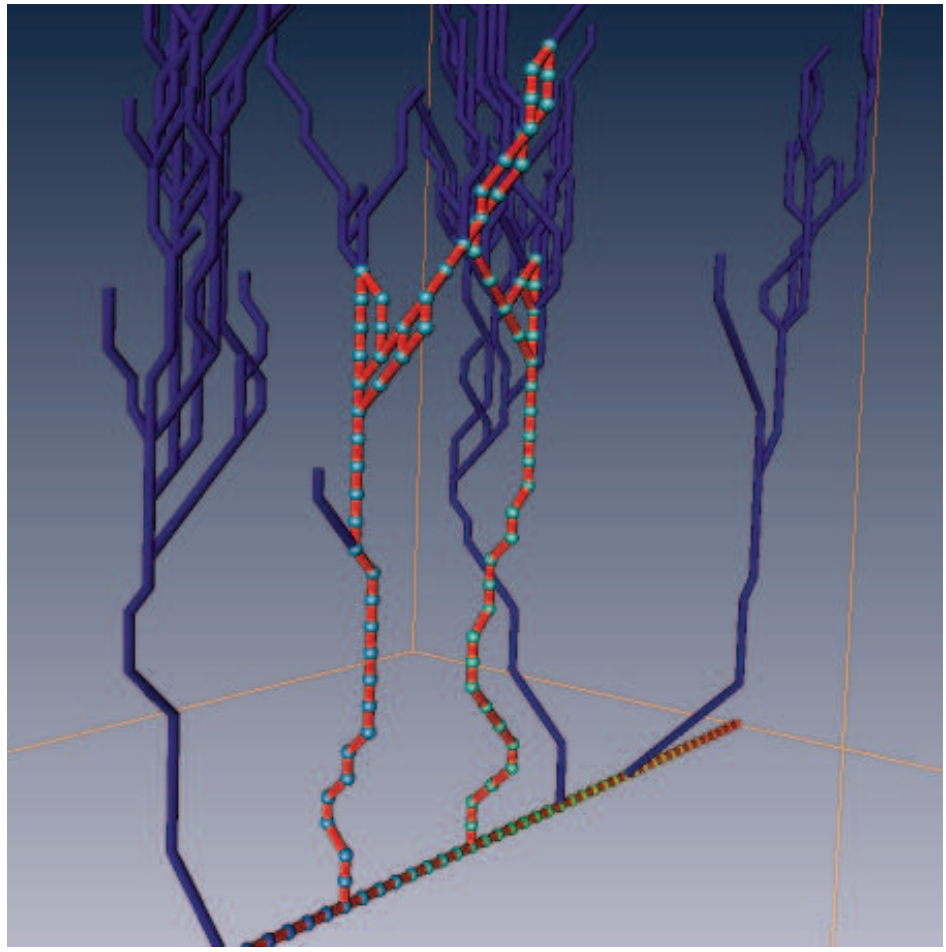

Fig. 10. Blood flow is calculated only in branches that created closed loops.

After upgrading graph structure the model calculates new flow distribution in network of vessel. It consist of three steps:

1. searching for closed loops in graph - blind sprouts are not take into consideration,

2. setting and solving system of equation for new pressures distribution in graph nodes,

3. calculating flows in graph according to pressures distribution

The framework are very flexible and we can use in the model 3D mesh (see Fig. 12). Hexagonal mesh can be also applied instead of rectangular (see Fig. 9). The model can be easily extended by new factors and processes. Figure 12 demonstrate configuration with source of angiogenic inhibitors which block vessel forming . 


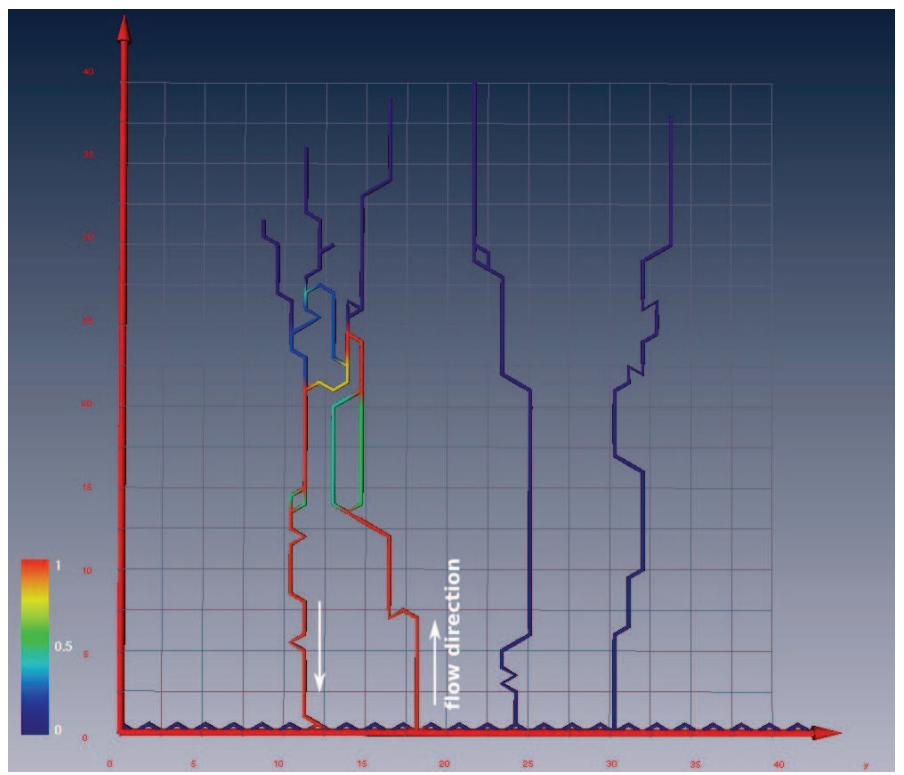

Fig. 11. Blood flow calculated in model of tumour induced angiogenesis. Vessels are coloured according to flow rate.

\section{Conclusions}

What is now what we called Cellular Automata? Initially Cellular Automata was treated rather as interesting mathematical model of complexity than tool for simulating complex physical phenomena. Their complex behaviour was the area of intensive and valuable studies on deterministic chaos and complexity. However when we try to reflect some real phenomena that classical definition appeared to be too limited. Some modifications were introduced to better encode modelled phenomena in Cellular Automata virtual "physics". Over time, modifications were becoming more serious. Is it still Cellular Automata? Let us enumerate what is in common in most models that use name "Cellular Automata":

- mesh, usually regular,

- evolution governed by rules,

- local neighbourhood (sometimes range is bigger then nearest cells),

It seems that those three features still are in common for models and modeling tools that use attribute "Cellular Automata".

Sometimes Cellular Automata models become very close to Agent-Based Models (ABM) approach (sometimes referred as multi-agent systems). However, it should be noticed that agents are characterized by much more complex and sophisticated behaviour of individual entities.

It is worth to emphasize that Cellular Automata paradigm its popularity as computer simulations and modelling tool partially owes the fact that it fits very well to computer implementation:

1. rules can be easily encoded in programming languages,

2. regular mesh of cells can be easily encoded in most typical data structures, 


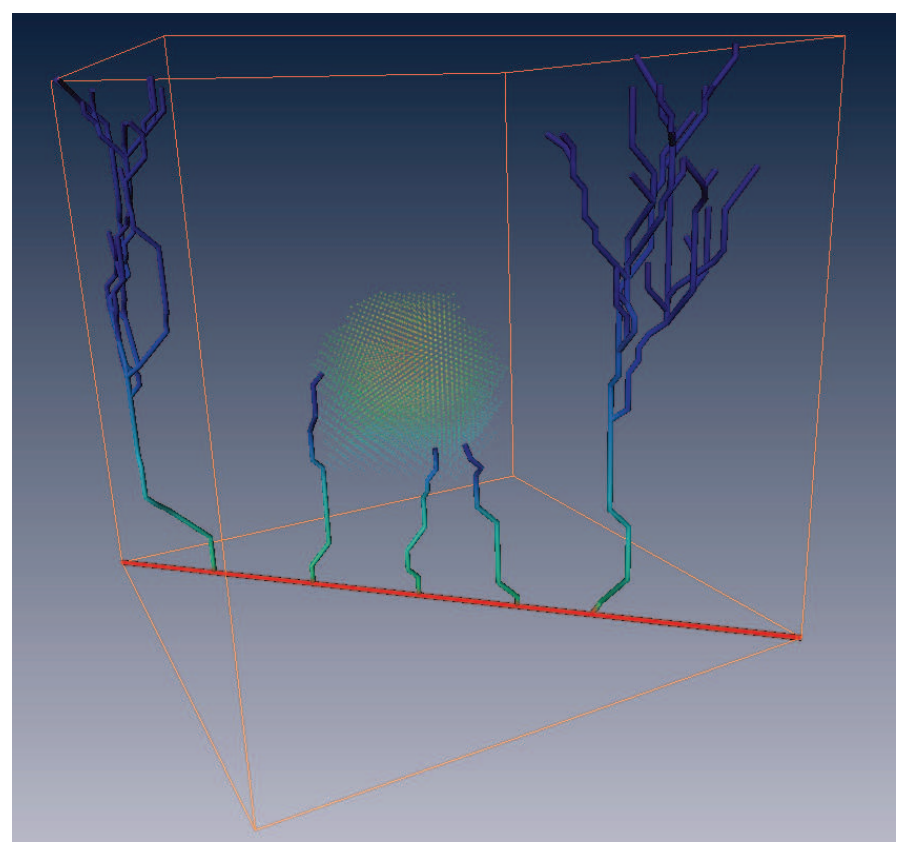

Fig. 12. Tumour induced angiogenesis in 3D. Cancer cells are located at the upper side of the box. Additionally sources of angiogenic inhibitors were located in the center of the box.

3. mesh and local neighbourhood eliminates laborious necessity of determining which elements interacts each other.

4. there is no round-off errors, numerical instabilities and so on...

In this chapter, the application of the Cellular Automata paradigm for modelling network systems was presented. The combination of Cellular Automata and graph structure was successfully applied for simulating phenomena that belong to general class of network systems located in consuming or producing environment. The examples show how broad meaning has Cellular Automata now. It should be also encouragement for further experiments with this useful paradigm that cannot be constrained by the definitions.

\section{Acknowledgements}

The author thanks to Witold Dzwinel (Institute of Computer Science, AGH University of Science and Technology) for his contribution to these works. The researches were partially supported by Institute of Computer Science, AGH University of Science and Technology (project no. 11.11.120.865) and also partially supported by the Polish Ministry of Education and Science (project no. NN 519579 338).

\section{References}

Baxter, G. \& Behringer, R. (1991). Cellular automata models for the flow of granular materials, Physica D: Nonlinear Phenomena 51(1-3): 465-471. 
URL: $h$ ttp://www.sciencedirect.com/science/article/B6TVK-46MV07J-19/2/ 058ea7eab54b1774410ee951bf6e69e9

Blue, V., Embrechts, M. \& Adler, J. (1997). Cellular automata modeling of pedestrian movements, Vol. 3, pp. 2320-2323 vol.3.

Cannataro, M., Gregorio, S. D., Rongo, R., Spataro, W., Spezzano, G. \& Talia, D. (1995). A parallel cellular automata environment on multicomputers for computational science, Parallel Computing 21(5): 803-823.

Carmeliet, P. (2005). Angiogenesis in life, disease and medicine, Nature 438(7070): 932-936. 10.1038 /nature04478.

Chopard, B. \& Droz, M. (1998). Cellular Automata Modeling of Physical Systems, Alea-Saclay Monographs and Textes in Statistical Physics, Cambridge University Press.

D'Ambrosio, D., Gregorio, S. D., Gabriele, S. \& Gaudio, R. (2001). A cellular automata model for soil erosion by water, Physics and Chemistry of the Earth, Part B: Hydrology, Oceans and Atmosphere 26(1): 33-39.

URL: http://www.sciencedirect.com/science/article/B6VPV-45KWJ8F-8/2/ e17522d634e01ce1769f19d5fe1cf975

Ferrara, N. \& Kerbel, R. S. (2005). Angiogenesis as a therapeutic target, Nature 438(7070): 967-974. 10.1038/nature04483.

Frisch, U., Hasslacher, B. \& Pomeau, Y. (1986). Lattice-gas automata for the navier-stokes equation, Phys. Rev. Lett. 56(14): 1505-1508.

Gobron, S., Altekin, A., Bonafos, H. \& Thalmann, D. (2010). Gpgpu computation and visualization of three-dimensional cellular automata, The Visual Computer 27: 67-81.

Gradzinski, R., Baryla, J., Doktor, M., Gmur, D., Gradzinski, M., Kedzior, A., Paszkowski, M., Soja, R., Zielinski, T. \& Zurek, S. (2003). Vegetation-controlled modern anastomosing system of the upper narew river (ne poland) and its sediments, Sedimentary Geology 157(3-4): 253-276.

Graner, F. \& Glazier, J. A. (1992). Simulation of biological cell sorting using a two-dimensional extended Potts model, Physical Review Letters 69: 2013-2016.

Hardy, J., de Pazzis, O. \& Pomeau, Y. (1976). Molecular dynamics of a classical lattice gas: Transport properties and time correlation functions, Phys. Rev. A 13(5): 1949-1961.

Kier, L. B. (2000). A cellular automata model of bond interactions among molecules, Journal of Chemical Information and Computer Sciences 40(5): 1285-1288.

Kier, L. B., Cheng, C.-K., Tute, M. \& Seybold, P. G. (1998). A cellular automata model of acid dissociation, Journal of Chemical Information and Computer Sciences 38(2): 271-275.

Margolus, N., Toffoli, T. \& Vichniac, G. (1986). Cellular-automata supercomputers for fluid-dynamics modeling, Phys. Rev. Lett. 56(16): 1694-1696.

Markus, M., Bohm, D. \& Schmick, M. (1999). Simulation of vessel morphogenesis using cellular automata, Mathematical Biosciences 156(1-2): 191-206.

URL: http://www.sciencedirect.com/science/article/B6VHX-3W374Y2-9/2/ d03620d18d402fe799635911fa3dab5c

Masselot, A. \& Chopard, B. (1996). Cellular automata modeling of snow transport by wind, in J. Dongarra, K. Madsen \& J. Wasniewski (eds), Applied Parallel Computing Computations in Physics, Chemistry and Engineering Science, Vol. 1041 of Lecture Notes in Computer Science, Springer Berlin / Heidelberg, pp. 429-435.

Mcdougall, S. R., Anderson, A. R. A., Chaplain, M. A. J. \& Sherratt, J. A. (2002). Mathematical modelling of flow through vascular networks: Implications for tumour-induced angiogenesis and chemotherapy strategies, Bulletin of Mathematical Biology 64(42): 673-702. 
Neumann, J. V. (1966). Theory of Self-Reproducing Automata, University of Illinois Press, Champaign, IL, USA.

Reis, E., Santos, L. \& Pinho, S. (2009). A cellular automata model for avascular solid tumor growth under the effect of therapy, Physica A: Statistical Mechanics and its Applications 388(7): 1303-1314.

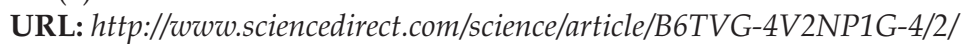
d7ec3cee53804a2bfa771c79ba7875bf

Somers, J. \& Rem, P. (1989). A parallel cellular automata implementation on a transputer network for the simulation of small scale fluid flow experiments, in G. van Zee \& J. van de Vorst (eds), Parallel Computing 1988, Vol. 384 of Lecture Notes in Computer Science, Springer Berlin / Heidelberg, pp. 116-126.

Steve, N. M., Webb, S. \& Othmer, H. G. (2004). Mathematical modeling of tumor-induced angiogenesis, J. Math. Biol 49: 111-187.

Toffoli, T. \& Margolus, N. (1987). Cellular automata machines: a new environment for modeling, MIT Press, Cambridge, MA, USA.

Tonini, T., Rossi, F. \& Claudio, P. P. (n.d.). Molecular basis of angiogenesis and cancer, Oncogene 22(42): 6549-6556.

Topa, P. (2006). Towards a two-scale cellular automata model of tumour-induced angiogenesis, in S. E. Yacoubi, B. Chopard \& S. Bandini (eds), ACRI, Vol. 4173 of Lecture Notes in Computer Science, Springer, pp. 337-346.

Topa, P. (2008). Dynamically reorganising vascular networks modelled using cellular automata approach, Cellular Automata, 8th International Conference on Cellular Automata for Reseach and Industry, ACRI 2008, Yokohama, Japan, September 23-26, 2008. Proceedings, Vol. 5191 of Lecture Notes in Computer Science, Springer, pp. 494-499.

Topa, P. \& Dzwinel, W. (2003). Consuming environment with transportation network modelled using graph of cellular automata, in R. Wyrzykowski, J. Dongarra, M. Paprzycki \& J. Wasniewski (eds), PPAM, Vol. 3019 of Lecture Notes in Computer Science, Springer, pp. 513-520.

Topa, P. \& Dzwinel, W. (2009). Using network descriptors for comparison of vascular systems created by tumour-induced angiogenesis, Theoretical and Applied Informatics 21(2): 83-94.

Topa, P., Dzwinel, W. \& Yuen, D. A. (2006). a Multiscale Cellular Automata Model for Simulating Complex Transportation Systems, International Journal of Modern Physics C 17: 1437-1459.

Topa, P. \& Paszkowski, M. (2001). Anastomosing transportation networks, in R. Wyrzykowski, J. Dongarra, M. Paprzycki \& J. Wasniewski (eds), PPAM, Vol. 2328 of Lecture Notes in Computer Science, Springer, pp. 904-912.

Wang, H., Nie, G. \& Fu, K. (2008). Cellular automata simulation of the growth of bone tissue, ICNC '08: Proceedings of the 2008 Fourth International Conference on Natural Computation, IEEE Computer Society, Washington, DC, USA, pp. 421-424.

Was, J. (2005). Cellular automata model of pedestrian dynamics for normal and evacuation conditions, ISDA '05: Proceedings of the 5th International Conference on Intelligent Systems Design and Applications, IEEE Computer Society, Washington, DC, USA, pp. 154-159.

Wolfram, S. (2002). A New Kind of Science, Wolfram Media.

URL: $h t t p: / / w w w . a m a z o n . c o m / e x e c / o b i d o s / A S I N / 1579550088 / \mathrm{ref}=$ nosim/rds-20

Wooldridge, M. (2009). An Introduction to Multiagent Systems, Wiley. 


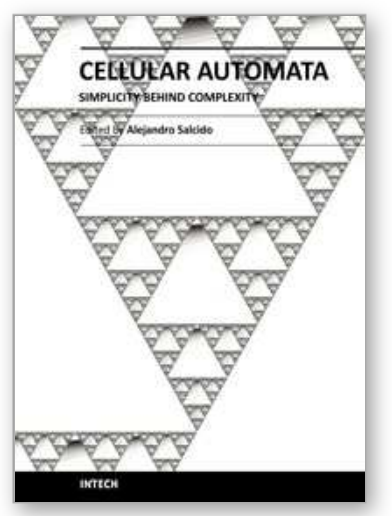

\author{
Cellular Automata - Simplicity Behind Complexity \\ Edited by Dr. Alejandro Salcido
}

ISBN 978-953-307-230-2

Hard cover, 566 pages

Publisher InTech

Published online 11, April, 2011

Published in print edition April, 2011

Cellular automata make up a class of completely discrete dynamical systems, which have became a core subject in the sciences of complexity due to their conceptual simplicity, easiness of implementation for computer simulation, and their ability to exhibit a wide variety of amazingly complex behavior. The feature of simplicity behind complexity of cellular automata has attracted the researchers' attention from a wide range of divergent fields of study of science, which extend from the exact disciplines of mathematical physics up to the social ones, and beyond. Numerous complex systems containing many discrete elements with local interactions have been and are being conveniently modelled as cellular automata. In this book, the versatility of cellular automata as models for a wide diversity of complex systems is underlined through the study of a number of outstanding problems using these innovative techniques for modelling and simulation.

\title{
How to reference
}

In order to correctly reference this scholarly work, feel free to copy and paste the following:

Pawel Topa (2011). Network Systems Modelled by Complex Cellular Automata Paradigm, Cellular Automata Simplicity Behind Complexity, Dr. Alejandro Salcido (Ed.), ISBN: 978-953-307-230-2, InTech, Available from: http://www.intechopen.com/books/cellular-automata-simplicity-behind-complexity/network-systems-modelledby-complex-cellular-automata-paradigm

\section{INTECH}

open science | open minds

\author{
InTech Europe \\ University Campus STeP Ri \\ Slavka Krautzeka 83/A \\ 51000 Rijeka, Croatia \\ Phone: +385 (51) 770447 \\ Fax: +385 (51) 686166 \\ www.intechopen.com
}

\author{
InTech China \\ Unit 405, Office Block, Hotel Equatorial Shanghai \\ No.65, Yan An Road (West), Shanghai, 200040, China \\ 中国上海市延安西路65号上海国际贵都大饭店办公楼405单元 \\ Phone: +86-21-62489820 \\ Fax: $+86-21-62489821$
}


(C) 2011 The Author(s). Licensee IntechOpen. This chapter is distributed under the terms of the Creative Commons Attribution-NonCommercialShareAlike-3.0 License, which permits use, distribution and reproduction for non-commercial purposes, provided the original is properly cited and derivative works building on this content are distributed under the same license. 